\title{
4 factors were predictive of extended duration in hospital in acute psychiatric admission
}

\author{
Hopko DR, Lachar D, Bailley SE, et al. Assessing predictive factors for extended hospitalization at acute psychiatric \\ admission. Psychiatr Serv 2001 Oct;52:1367-73.
}

\section{QUESTION: How accurate is information obtained early in the process of admission to hospital for identifying acute psychiatric admissions that will require extended care?}

\section{Design}

2 cohort studies: 1 for derivation and 1 for validation of the clinical prediction model results.

\section{Setting}

University affiliated psychiatric hospital in Houston, Texas, USA.

\section{Patients}

2430 acute psychiatric inpatients (mean age 38 y, 56\% men) randomly assigned to the derivation and validation subsets (1215 patients in each group).

\section{Description of prediction guide}

Data were collected on demographic characteristics and history of previous admissions to hospital. The Brief Psychiatric Rating Scale-Anchored Version (BPRS-A) was completed by attending faculty psychiatrists to describe patients within 24 hours of admission, and total score and 4 new subscales derived from these ratings were calculated*.

Source of funding:

Harris County

Psychiatric Center.

For correspondence: Dr D Lachar,

University of Texas

Houston Medical

School, Houston, Texas,

USA.

david.lachar@uth.tmc.edu

\section{Main outcome measures}

Discharge to the community or transported to another hospital for extended care.

\section{Main results}

6 factors were found to be predictive of discharge status across both the derivation and validation subsets of patients (marital status, number of previous admissions to the psychiatric hospital, number of previous transfers for extended care, and scores on the BPRS-A subscales for resistance, positive symptoms, and psychological discomfort). These 6 variables were examined through stepwise discriminant function analysis on the derivation sample. All variables except the number of previous admissions to the psychiatric hospital were retained. 4 of the variables were replicated in an identical analysis done on the validation sample. The discriminant coefficients were BPRS-A resistance score (0.48), BPRS-A positive symptoms score $(0.45)$, previous referrals for extended care (0.44), and BPRS-A psychological discomfort score $(-0.30)$. A final discriminant analysis on the entire sample, which included the 4 predictor variables, showed a classification accuracy of $70 \%$ for extended duration in hospital and $80 \%$ for community discharge. Overall the classification accuracy of this model was $78 \%$.

\section{Conclusion}

In acute psychiatric admissions, 4 factors were predictive of extended duration in hospital; Brief Psychiatric Rating Scale-Anchored Version (BPRS-A) resistance score, BPRS-A positive symptoms score, previous referrals for extended care, and BPRS-A psychological discomfort score.

*See: Lachar D, Bailley SE, Rhoades HM, et al. New subscales for an anchored version of the Brief Psychiatric Rating Scale: construction, reliability, and validity in acute psychiatric admissions. Psychol Assess 2001;13:384-95.

\section{COMMENTARY}

A clinically important task is the identification of patients at admission, in the hope that more appropriate care can be offered and prolonged admission avoided. Previous research to identify clinically useful factors that predict high use of psychiatric services has not been fruitful. Hopko et al present a well conducted cohort study to identify such factors. Scores on the clinician rated BPRS seem to be predictive, and were replicated in a concurrent, randomly selected cohort.

How useful are these findings in clinical practice? The research of Hopko et al is an interesting observational investigation, but it raises questions about its clinical significance. Firstly, we need to know the positive predictive value of BPRS scores in the prospective identification of those at risk of prolonged admission. It will vary for different healthcare settings and for different populations, and may preclude the utility of BPRS scores. Secondly, we need to know whether identifying such patients really does improve their care and outcome. The appropriate design to evaluate this question is a randomised trial, ${ }^{1}$ whereby clinical teams are randomly assigned to have BPRS scores for individual patients assessed at admission and fed back into routine care planning. The findings of any such research have yet to be reported.

There are reasons to be cautious about the utility of incorporating symptom rating scales into clinical decision making. Firstly, clinicians are generally reluctant to collect complex psychometric scales for each of their patients. ${ }^{2}$ Secondly, research examining the potential of routinely administered psychiatric instruments to improve patient care and outcome in non-specialist settings has been negative. ${ }^{3}$

It takes time and resources to administer, score, and feed back the results of complex questionnaires for each patient. Sufficient benefit should be shown to justify this effort, because it represents an opportunity cost. In the absence of this evidence, the routine administration of questionnaires such as the BPRS is, as yet, an unproven technology.

Simon Gilbody, MBChB, DPhil, MRCPsych University of Leeds Leeds, UK

1 Donner A. Cluster randomisation trials in mental health research [EBMH notebook]. Evidence-Based Mental Health 2000;3:102-3.

2 Gilbody SM, House AO, Sheldon TA. UK psychiatrists do not use outcomes measures - a national survey. Br J Psychiatry 2002;180:101-3.

3 Gilbody SM, House AO, Sheldon TA. Routinely administered questionnaires for depression and anxiety: systematic review. BMJ 2001;322:406-9. 